\title{
The importance of IL- 6 protein expression in primary human renal cell carcinoma: an immunohistochemical study
}

Bernard Paule, Jacqueline Belot, Christiane Rudant, Colette Coulombel, Claude Clément Abbou

\begin{abstract}
Aims-Interleukin 6 (IL-6) is produced by some renal carcinoma cell lines in vitro. This might be biologically important because IL-6 is a cytokine of particular interest, owing to its involvement in the growth of renal cell carcinoma. In this study, the expression of IL-6 protein in tissue samples from primary renal cell carcinoma was analysed, and then its clinical importance was examined.

Methods-The distribution of IL-6 in renal cell carcinoma was examined by means of an immunohistochemical method in 47 untreated primary renal cell carcinoma samples. The search for a significant difference between histological patterns, Furhman's grading system, TNM classification, and IL-6 protein ex-
\end{abstract} pression was carried out.

Results-Immunohistochemistry demonstrated that IL-6 is expressed in $70 \%$ of primary tumours. There was no significant difference in the tumour size and grade between renal cell carcinomas with or without IL-6 expression. However, a relatively large number of high grade tumours expressed IL-6.

Conclusion-The importance of IL-6 expression with regard to tumour size/local growth is questionable because IL-6 has been correlated with the development of metastatic disease. These data suggest that the production of IL-6 could exert a growth inhibitory effect on primary renal cell carcinoma.

(F Clin Pathol 2000;53:388-390)

Keywords: interleukin 6; renal cell carcinoma; immunohistochemistry

Interleukin 6 (IL-6) is produced by some renal carcinoma cell lines in vitro. ${ }^{1}$ IL- 6 has been detected in the serum of patients with advanced renal cell carcinoma. ${ }^{2}{ }^{3}$ Experimental evidence has shown that IL- 6 could act as an autocrine growth factor in human renal cell carcinoma. ${ }^{45}$ More recently, Takenawa and colleagues ${ }^{6}$ showed that $26 \%$ of primary renal cell carcinomas express IL- 6 protein and IL-6 receptor transcripts. These results suggest that IL- 6 could contribute to the development or progression of tumour growth. In our study, we analysed IL-6 protein expression with an immunohistochemical staining method in tissue samples from primary renal cell carcinoma.
The clinical importance of IL-6 protein expression was then examined.

\section{Patients and methods}

PATIENTS AND SAMPLES

Our study was carried out on 47 patients with untreated primary renal cell carcinoma. All patients (27 men, 20 women) with renal cell carcinoma were treated with radical nephrectomy between 1995 and 1997. No patient had detectable tumour metastases at the time of surgery. The age of the patients ranged from 22 to 78 years (mean age, 58). After nephrectomy, each tumour was classified according to the histological type as reviewed by Weiss and colleagues $^{7}$ and Storkel and colleagues ${ }^{8}$ : the TNM staging classification of the International Union against Cancer 1992 and the grading system of Furhrman. ${ }^{9}$

\section{IMMUNOCHEMICAL ANALYSIS}

IL-6 expression was evaluated on 47 untreated primary renal cell carcinoma samples by means of immunohistochemistry. Immunohistochemical analysis was performed on the primary renal tumours. Tissue specimens were fixed in AFA (alcohol/formalin/acetone) and paraffin wax embedded. Tissue sections $(5 \mu \mathrm{m}$ thick) were cut on super Frost/P microscope slides (Menzel-Glaser, Templemars, Paris, France) and dewaxed by three 10 minute treatments in LMR-sol (Labo-Moderne, Paris, France). The sections were rehydrated by two five minute incubations in a graded series of alcohol (100\%, 90\% and $70 \%$ ethanol). The sections were rinsed in $0.1 \mathrm{M}$ phosphate buffered saline (PBS). We used the biotin blocking system kit (Dako, Trappes, France) to inhibit non-specific sites as a result of endogenous biotin. After washing with 0.1 M PBS, the non-specific sites were blocked by incubation with goat serum in PBS for 20 minutes at room temperature. The sections were then incubated with monoclonal mouse antihuman IL-6 (Genzyme, St Christoph, France) at a concentration of $50 \mathrm{ng} / \mathrm{ml}$. After an overnight incubation at $4^{\circ} \mathrm{C}$, the slides were washed three times with PBS and incubated for 30 minutes with biotinylated antimouse immunoglobulin (LSAB kit peroxidase; Dako). Slides were washed with PBS and endogenous peroxidase activity was quenched by incubating the sections for 10 minutes with 3\% hydrogen peroxide. After three five minute washes in PBS, the sections were incubated for 10 minutes at room temperature with peroxidase labelled 


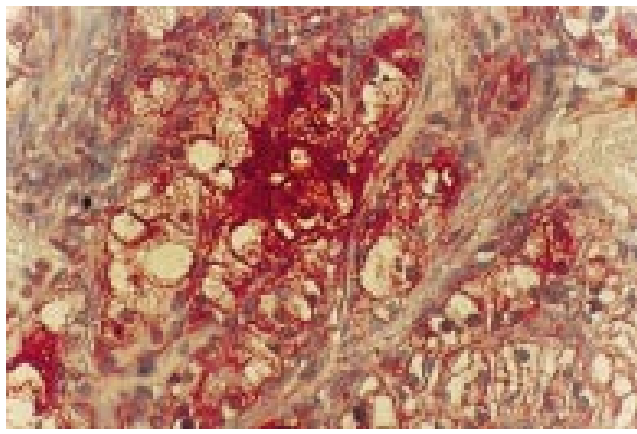

Figure 1 Interleukin 6 (IL-6) specific immunostaining of clear cell carcinoma (original magnification $\times 400$ ).

streptavidin (LSAB kit peroxidase; Dako). The peroxidase reaction was performed using AEC chromogen $(3 \%, 3$ '-amino-9'-ethylcarbazol in $\mathrm{N}, \mathrm{N}$-dimethylformamide). After the staining reaction, the sections were counterstained with Mayer's haematoxylin. Staining was assessed by two independent observers. Each specimen was then reviewed by a pathologist to confirm the histological diagnosis and the specificity of staining. For each specimen, two sections were studied by immunochemistry. A negative tumour control for IL-6 expression was performed on normal tissue adjacent to the tumour, and was always negative for IL- 6 . The variation in staining intensity was defined qualitatively by the most abundant staining pattern for each specimen and was graded on a 5 point scale ( 0 to $4+$ ).

STATISTICAL ANALYSIS

The search for a significant difference between histological patterns, Furhrman's grading system, the TNM classification, and IL- 6 protein expression was carried out. Ratios were compared through $\chi^{2}$ tests without continuity correction and Fisher's exact test.

\section{Results}

Thirty three of $47(70 \%)$ samples were positive for IL-6 immunoreactivity. The neoplastic cells showed cytoplasmic staining for IL-6. In the case of positive staining, $27 \%$ of the samples had a staining intensity score of $1+, 8 \%$ scored $2+, 21 \%$ scored $3+$, and $17 \%$ scored $4+$. A diffuse infiltration of neoplastic cells positive for IL-6 was seen in each section. All the samples from poorly differentiated carcinomas stained with $3+$ or $4+$ intensity.

Twenty eight of 37 clear cell carcinomas, one chromophobe carcinoma, and four of nine papillary carcinomas were found to be immunoreactive with the anti-IL-6 monoclonal antibody (fig 1). No correlation was found between IL-6 protein expression and tumour grade (G1-G2 v G3-G4; p = 0.09; Fisher's exact test), stage (pT1-pT2 v pT3-pT4; p = 0.025; Fisher's exact test) or histological type $\left(p=0.92 ; \chi^{2}\right.$ test $)$. However, a relatively large number (13 of 14) of high nuclear grade tumours were immunoreactive for IL-6 expression. During this period, two patients with grade 3 tumours died because of multiple metastases. Their tumoral cells had a staining intensity of $4+$.

\section{Discussion}

Our immunohistochemical analysis found that IL-6 was expressed in $70 \%$ of primary renal cell carcinomas. Recently, Takenawa et al detected IL- 6 gene expression in five of seven tumour cell lines and in 22 of 43 primary renal cell carcinoma tissues. ${ }^{6} \mathrm{~A}$ specific transcript of the IL-6 receptor in $11(26 \%)$ of 43 primary renal cell carcinomas was identified. Immunohistochemical analysis confirmed the expression of IL- 6 by the tumour cells. These results demonstrated the presence of an autocrine mechanism in renal cell carcinoma. There was no significant difference in the tumour size or grade between renal cell carcinomas with or without expression of IL-6. In that study, the correlation with histological patterns was not examined. In our study, there were no significant differences between IL- 6 expression and tumour stage, histological type, or nuclear grade, but relatively more high grade tumours expressed IL-6. This finding is of interest because IL-6 expression has been correlated with shorter survival and immunotherapy resistance; in addition, the nuclear grading $(1-2 v 3-4)$ is generally considered to be a significant prognostic determinant in the development of systemic disease.

IL-6 production has been seen in renal carcinoma cell lines in vitro, ${ }^{16}$ and has been shown to induce tumour cell growth. In view of the autocrine growth factor role ascribed to IL-6 in cell lines, ${ }^{45}$ our data suggest that IL-6 could exert a growth stimulatory effect on some primary renal cell carcinomas. However, IL-6 expression is likely to be related to metastatic disease (that is, lymph node metastasis or distant organ metastasis). ${ }^{10}$ Costes et al have shown that most patients with metastases have some IL-6 receptor positive tumour cells. ${ }^{11}$ In contrast, no strictly intrarenal tumours showed positive staining. Interestingly, most grade 3 tumours were positive for IL-6 receptor immunostaining. Therefore, the importance of IL-6 expression with regard to tumour size and local growth (confined within or locally extending beyond the kidney) is questionable. The increased expression of IL-6 might reflect a true upregulation but no growth advantage. Lu et al showed that the growth of melanoma cells obtained from early stage primary tumours is inhibited by IL- $6 .{ }^{12}$ This growth inhibitory effect was lost in the more advanced stage derived cell lines. Similarly, the production of IL- 6 could exert a growth inhibitory effect on primary renal cell carcinomas without metastases, and a growth stimulatory effect in systemic disease.

Cytokine signals might be important in the potential biological effects of IL- 6 . The production of IL-6 is regulated by cytokine signals. In renal carcinoma cell lines, tumour necrosis factor upregulated the production of IL-6, with synergistic action by interferon $\gamma \cdot{ }^{13}$ Conversely, in non-producers of IL-6, transforming growth factor $\beta$ or other cytokines could inhibit the production of endogenous IL-6. ${ }^{14}{ }^{15}$

In our study, we have shown that IL-6 protein can be detected by immunohistochemistry in $70 \%$ of primary renal cell carcinomas. 
The importance of IL- 6 expression with regard to tumour size/local growth is intriguing because IL- 6 has been correlated with the development of metastatic disease. In primary renal cell carcinomas, the increased expression of IL- 6 might reflect no growth advantage. It is interesting to speculate on whether this increased expression of IL-6 in high grade tumours is directly responsible for their progression or merely indicative of an increasing deregulation of cell cycle control because nuclear grading is generally considered to be an important prognostic determinant in the development of systemic disease.

The role of IL-6 is likely to be complex because various cytokines might influence each other, and regulatory mechanisms at the genetic level might also be involved in growth factor expression.

1 Koo AS, Armstrong C, Bochner B, et al. Interleukin 6 and renal cancer production, regulation and growth effects. Cancer Immunol Immunother 1992;35:97-105.

2 Blay JM, Negrier S, Combaret V, et al. Serum level of interleukin 6 as a prognostic factor in metastatic renal cell carcinoma. Cancer Res 1992;52:3317-22.

3 Tsukamaoto T, Kumamoto Y, Miyao N, et al. Interleukin-6 in renal cell carcinoma. f Urol 1992;148:1778-82.

4 Blay JY, Bolon J, Alberte I, et al. Growth inhibition of renal carcinoma cell lines by interleukin 6 antisense oligonucleotides: an internal autocrine loop [abstract]. Pro-
ceedings of the American Association of Clinical Research ceedings of the

5 Miki S, Iwano M, Miki Y. Interleukin-6 (IL-6) functions as an in vitro autocrine growth factor in renal cell carcinoma. FEBS Lett 1989;250:607-10

6 Takenawa J, Kanako Y, Fukijumoto M, et al. Enhanced expression of interleukin- 6 in primary human renal cell carcinomas. F Natl Cancer Inst 1991;83:1668-72.

7 Weiss LM, Gelb AB, Medeiros LJ. Adult renal epithelial neoplasms. Am f Clin Pathol 1995;103:624-35.

8 Storkel S, Eble J, Adlaka K, et al. Morphological classification of renal cell carcinoma. Cancer 1997;80:95587.

9 Furhrman SA, Lasky LC, Limas C. Prognostic significance of morphologic parameters in renal cell carcinoma. Am $\mathcal{f}$ Surg Pathol 1982;6:655-63.

10 Paule B, Clerc D, Rudant C, et al. Enhanced expression of interleukin-6 protein in bone and serum of metastatic renal interleukin-6 protein in bone and serum of met

11 Costes V, Liautard J, Pico MC, et al. Expression of the interCostes V, Liautard J, Pico MC, et al. Expression of the inter-
leukin 6 receptor in primary renal cell carcinoma. $f$ Clin Pathol 1997;50:835-40.

$12 \mathrm{Lu}$ C, Vickers MF, Kerbel RS. Interleukin 6: a fibroblastderived growth inhibitor of human melanoma cells from early but not advanced stages of tumour progression. Proc Natl Acad Sci U S A 1992;89:9215-19.

13 Koo AS, Bochner B, de Kermion JB, et al. Production and regulation of interleukin- 6 by renal cell carcinoma in vitro: protein activity and mRNA gene expression. Surg Forum 1990;41:710-13.

14 Lagneaux L, Delforge A, Dorval C, et al. Excessive production of transforming growth factor $\beta$ by bone marrow stroma cells in $\mathrm{B}$ cell chronic lymphocytic leukemia inhibits growth of hematopoietic precursors and interleukin 6 production. Blood 1993;82:2379-85.

15 Lotem J, Sachs L. Hematopoietic cytokines inhibit apoptosis induced by transforming growth factor $\beta$ and cancer chemotherapy compounds in myeloid leukemic cells. Blood 1992;50:1750-4. 